\title{
Case Report \\ Isolated Central Nervous System Relapse in Chronic Myeloid Leukemia
}

\author{
Juliana Gomez $^{1}$ and Victor Duenas ${ }^{2}$ \\ ${ }^{1}$ Internal Medicine Department, Guillermo Almenara Irigoyen National Hospital, Grau Avenue 800, Lima, Peru \\ ${ }^{2}$ National Institute of Neurologic Sciences, Ancash Street 1271, Barrios Altos, Lima, Peru \\ Correspondence should be addressed to Juliana Gomez; juliana_gab@yahoo.com
}

Received 23 October 2014; Revised 8 March 2015; Accepted 9 March 2015

Academic Editor: Lothar Bergmann

Copyright (C) $2015 \mathrm{~J}$. Gomez and V. Duenas. This is an open access article distributed under the Creative Commons Attribution License, which permits unrestricted use, distribution, and reproduction in any medium, provided the original work is properly cited.

Chronic myeloid leukemia is a myeloproliferative disorder that has three distinguished phases: chronic, accelerated, and blastic. In extremely rare cases, the blast phase can affect the central nervous system without concomitant bone marrow involvement. We report the case of a patient with chronic myeloid leukemia who, despite having achieved complete cytogenetic remission in the bone marrow for several years, experienced a blast crisis of the central nervous system following an episode of infectious meningoencephalitis.

\section{Introduction}

Chronic myeloid leukemia (CML) is a myeloproliferative disorder characterized by the presence of a reciprocal translocation between chromosomes 9 and 22, known as the Philadelphia chromosome, which results in expression of an oncoprotein, termed BCR-ABL $[1,2]$. CML has 3 phases: chronic, accelerated, and blast phase [3]. Blast phase (BP) or blast crisis is defined as the presence of $20 \%$ or more blasts in peripheral blood or bone marrow (BM), or a large focus of blasts in BM, or an extramedullary blast proliferation [4]. The central nervous system as a site of extramedullary blast crisis is extremely rare [5], and when affected it usually occurs concurrently with systemic relapse [6].

We here describe the case of a patient with chronic myeloid leukemia who, after an episode of infectious meningoencephalitis, experienced blast crisis of the central nervous system although having achieved complete cytogenetic remission in the bone marrow for several years with imatinib treatment.

\section{Case Presentation}

The patient was a 33-year-old Hispanic man who had a history of Philadelphia-positive CML, diagnosed in the chronic phase 5 years ago. After diagnosis, he received treatment with imatinib $400 \mathrm{mg}$ QD, demonstrating complete hematological and cytogenetic bone marrow response within 4 months of therapy initiation. This treatment was maintained in the following years without relapse. The patient presented to the emergency department having experienced an eight-day history of severe headaches, nausea, and confusion and 3 days of low-grade fever. Upon direct examination the patient was febrile $\left(100.4^{\circ} \mathrm{F}\right)$, tachycardic, and lethargic, showed nuchal rigidity, and had 3 episodes of tonic-clonic seizures. The patient did not present with evidence of lymphadenopathy or hepatosplenomegaly. Head and thoracoabdominopelvic computed tomography scans were normal. WBC count was $12960 / \mu \mathrm{L}$ (75\% neutrophils, 3\% bands, 4\% monocytes, and $18 \%$ lymphocytes). CSF showed 1010 cells/ $\mu \mathrm{L}$ (100\% mononuclears), glucose of $41 \mathrm{mg} / \mathrm{dL}$ (N: 40-76), and proteins of $29 \mathrm{mg} / \mathrm{dL}$ (N: 15-45); Gram stain and HIV antibody detection were negative. PCR testing of the CSF and viral culture for herpes simplex were not available at our institution. The patient received treatment with ceftriaxone, vancomycin, acyclovir, mannitol, and anticonvulsants. Imatinib was discontinued thereafter. Five days later the patient's symptoms improved, he was alert, oriented, and afebrile, and the leukocytosis resolved. He subsequently was managed 

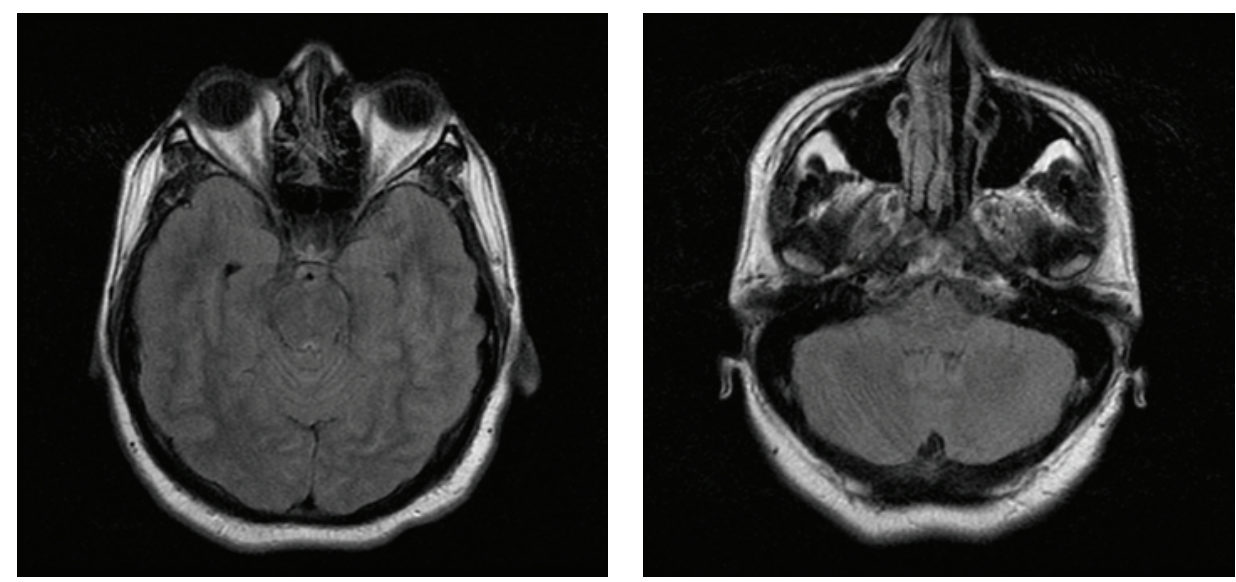

FIGURE 1: Brain MRI in FLAIR sequence, without abnormalities.
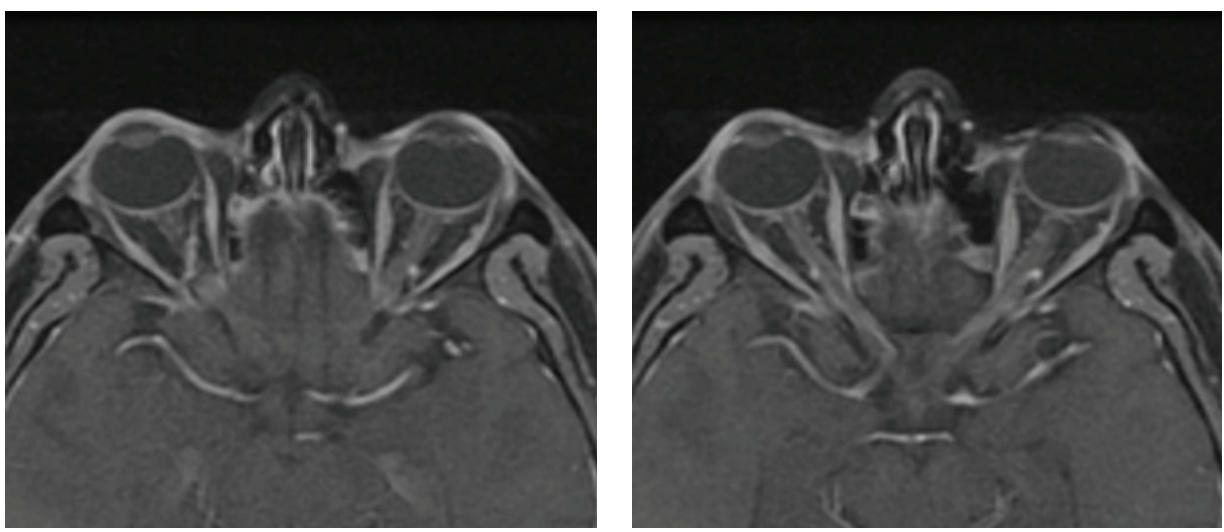

FIgURE 2: Optic MRI without abnormalities.

with acyclovir and anticonvulsants after obtaining negative bacterial CSF and blood cultures.

Eighteen days after admission the patient again developed severe headaches, vomit, confusion, and worse nuchal rigidity. A brain magnetic resonance imaging (MRI) scan was normal (Figure 1). BM aspiration assessment indicated complete hematologic, cytogenetic, and molecular remission. New CSF study showed 760 cells/ $\mu \mathrm{L}$ (100\% mononuclears), glucose of $24 \mathrm{mg} / \mathrm{dL}$, and proteins of $79 \mathrm{mg} / \mathrm{dL}$, and cytocentrifuge preparations lead to cytological identification of leukemic blasts.

CSF cytogenetic study showed 46XY, t(9;22)(q34:q11). Fluorescence in situ hybridization (FISH) analysis detected BCR-ABL fusion signals in $4.1 \%$ International Scale (IS) of cells. Flow cytometry to determine the blast type and measurement of imatinib concentration in serum and CSF were not available at our institution at the time. One month following admission, the patient received intrathecal methotrexate $(12 \mathrm{mg})$, cytosine arabinoside $(30 \mathrm{mg})$, and dexamethasone ( $4 \mathrm{mg}$ ), a total of 4 doses in a month. Few days after the first dose, the neurological symptoms improved substantially and the patient was able to initiate dasatinib $70 \mathrm{mg}$ BID PO. However, when fully oriented, the patient manifested decreased visual acuity. Funduscopy showed pale discs without edema suggestive of optic nerve atrophy. Optic MRI did not evidence abnormalities (Figure 2). After a few weeks the patient achieved full recovery except for the persistence of severe visual deficit. CSF became negative for blasts after 2 weeks of intrathecal therapy. The patient was discharged on dasatinib treatment. Follow-up at 6 months after discharge indicated the patient remained in complete cytogenetic response without signs of systemic or central nervous system (CNS) relapses; unfortunately, the severe visual impairment persisted.

\section{Discussion}

Chronic myeloid leukemia is a myeloproliferative neoplasm with an incidence of one-two cases per 100000 adults and accounts for $15 \%$ of newly diagnosed cases of leukemia in adults in the USA [7]. About 30 to $50 \%$ of CML patients are asymptomatic at diagnosis, in whom the disease is found on routine physical examination or blood tests. CML can be classified into chronic phase, accelerated phase, and blast phase. Diagnosis is most commonly made during the chronic stage and, if present, the symptomatology of this phase is mainly the result from anemia and splenomegaly. Lymphadenopathy, constitutional symptoms, and infiltration 
of skin or other tissues are uncommon. When present, they favor Philadelphia-negative CML or accelerated phase or blast phase of CML. Accelerated phase might be insidious or present with worsening anemia, splenomegaly, and organ infiltration. Most patients evolve into accelerated phase before BP, but $20 \%$ transit directly into $\mathrm{BP}$, which usually presents with worsening constitutional symptoms, bleeding, fever, and infections [3]. In $5-10 \%$ of the cases, the blast phase can present at extramedullary sites [2]. Lymph nodes, serosal surfaces, skin and soft tissue, breast, bone, and gastrointestinal or genitourinary tract are among the principal areas involved [8]. The central nervous system as a site of extramedullary blast crisis is quite rare [5].

In previously reported cases of CML with isolated CNS blast crisis, the main presenting symptom was headache with or without other neurologic symptoms. The majority of patients had complete cytogenetic response state at CNS relapse and the median time from treatment initiation to CNS relapse was 24 months with a range of 3 to 58 months [5, 822].

Imatinib, a BCR-ABL tyrosine kinase inhibitor, is capable of inducing major or complete cytogenetic responses in most CML patients $[23,24]$, changing dramatically the 10-year overall survival from 20 to $80-90 \%$ [7, 25]; however, it has been documented that it penetrates poorly into the CSF [6, $11,26]$, probably due to increased efflux of the drug from the CNS due to P-glycoprotein [27]. This emphasizes the problem of imatinib pharmacokinetics in the CNS, which might be an important issue to consider in patients taking the drug for over a year [12].

It has been suggested that CML patients with complete cytogenetic response following imatinib regimen and with history of any other CNS disease may need prophylaxis against CML expansion in CNS. Isobe et al. reported a case similar to ours of a male patient with CML in complete cytogenetic response for less than a year who developed CNS relapse after discontinuation of imatinib intake for over 6 weeks due to complications of viral meningitis [11]; however, our patient was in remission for over 4 years and the discontinuation of the drug was around 3 weeks when he developed symptoms of CNS relapse. Identifying which conditions may increase the risk of CNS involvement after receiving imatinib therapy in CML patients could be very helpful in the future [10, 11].

In the last decade there have been several reports of CML patients with CNS BP while on imatinib therapy; however, no surveillance strategy for CNS relapse or treatment guidelines for documented CNS relapse in this kind of patients have yet been defined.

Although most cases of CNS BP have been treated with triple intrathecal chemotherapy $[5,8,9,12,15]$, dasatinib, a second generation multityrosine kinase inhibitor with much greater potency (325-fold) over imatinib [28, 29], has also been used, in addition to chemotherapy, for that purpose in some cases reported, as well as for maintenance treatment, with good outcomes $[9,10,14,15,28]$.

Unfortunately, our patient was left with significant visual sequela, probably due to chronic intracranial hypertension and/or optic nerve leukemic infiltration; if CNS BP had been suspected and diagnosed earlier, perhaps this complication could have been prevented or properly treated. This highlights the fact that physicians must have a high suspicion index for CNS infiltration in patients with a CML history who manifest headache and meningismus even after achieving complete cytogenetic and molecular BM response with imatinib.

\section{Ethical Approval}

Informed consent was obtained from the patient for publication of this case report.

\section{Conflict of Interests}

The authors state that there is no conflict of interests.

\section{References}

[1] J. D. Rowley, "A new consistent chromosomal abnormality in chronic myelogenous leukaemia identified by quinacrine fluorescence and Giemsa staining," Nature, vol. 243, no. 5405, pp. 290-293, 1973.

[2] D. Eric and M. Hsi, Hematopathology - A Volume in Foundations in Diagnostic Pathology Series, Philadelphia Churchill Livingstone, 1st edition, 2006.

[3] E. Jabbour and H. Kantarjian, "Chronic myeloid leukemia: 2012 update on diagnosis, monitoring, and management," American Journal of Hematology, vol. 87, no. 11, pp. 1037-1045, 2012.

[4] S. H. Swerdlow, E. Campo, N. L. Harris et al., WHO Classification of Tumours of Haemotopietic and Lymphoid Tissues, IARC Press, Lyon, France, 4th edition, 2008.

[5] S. Rajappa, S. G. Uppin, D. Raghunadharao, I. S. Rao, and A. Surath, "Isolated central nervous system blast crisis in chronic myeloid leukemia," Hematological Oncology, vol. 22, no. 4, pp. 179-181, 2004.

[6] H. Pfeifer, B. Wassmann, W.-K. Hofmann et al., "Risk and prognosis of central nervous system leukemia in patients with philadelphia chromosome-positive acute leukemias treated with imatinib mesylate," Clinical Cancer Research, vol. 9, no. 13, pp. 4674-4681, 2003.

[7] A. Jemal, R. Siegel, J. Xu, and E. Ward, “Cancer statistics, 2010," CA: Cancer Journal for Clinicians, vol. 60, no. 5, pp. 277-300, 2010.

[8] A. Altintas, T. T. Cil, I. Kilinc, M. A. Kaplan, and O. Ayyildiz, "Central nervous system blastic crisis in chronic myeloid leukemia on imatinib mesylate therapy: a case report," Journal of Neuro-Oncology, vol. 84, no. 1, pp. 103-105, 2007.

[9] M. J. Park, P. H. Park, Y. H. Seo et al., "A case of isolated lymphoblastic relapse of the central nervous system in a patient with chronic myelogenous leukemia treated with imatinib," Annals of Laboratory Medicine, vol. 34, no. 3, pp. 247-251, 2014.

[10] S. M. Lindhorst, R. D. Lopez, and R. D. Sanders, "An unusual presentation of chronic myelogenous leukemia: a review of isolated central nervous system relapse," Journal of the National Comprehensive Cancer Network, vol. 11, no. 7, pp. 745-750, 2013.

[11] Y. Isobe, K. Sugimoto, A. Masuda, Y. Hamano, and K. Oshimi, "Central nervous system is a sanctuary site for chronic myelogenous leukaemia treated with imatinib mesylate," Internal Medicine Journal, vol. 39, no. 6, pp. 408-411, 2009. 
[12] M. Bornhauser, A. Jenke, J. Freiberg-Richter et al., "CNS blast crisis of chronic myelogenous leukemia in a patient with a major cytogenetic response in bone marrow associated with low levels imatinib mesylate and its $\mathrm{N}$-desmethylated metabolite in cerebral spinal fluid," Annals of Hematology, vol. 83, no. 6, pp. 401-402, 2004.

[13] M. E. Rytting and W. G. Wierda, "Central nervous system relapse in two patients with chronic myelogenous leukemia in myeloid blastic phase on imatinib mesylate therapy," Leukemia \& Lymphoma, vol. 45, no. 8, pp. 1623-1626, 2004.

[14] K. J. Aichberger, S. Herndlhofer, H. Agis et al., "Liposomal cytarabine for treatment of myeloid central nervous system relapse in chronic myeloid leukaemia occurring during imatinib therapy," European Journal of Clinical Investigation, vol. 37, no. 10, pp. 808-813, 2007.

[15] M. Nishimoto, H. Nakamae, K.-R. Koh et al., "Dasatinib maintenance therapy after allogeneic hematopoietic stem cell transplantation for an isolated central nervous system blast crisis in chronic myelogenous leukemia," Acta Haematologica, vol. 130, no. 2, pp. 111-114, 2013.

[16] N. A. Johnson, R. Fetni, and S. N. Caplan, "Isolated central nervous system relapse in patients with chronic myeloid leukemia on imatinib mesylate," Leukemia and Lymphoma, vol. 46, no. 4, pp. 629-630, 2005.

[17] M. Matsuda, Y. Morita, T. Shimada et al., "Extramedullary blast crisis derived from 2 different clones in the central nervous system and neck during complete cytogenetic remission of chronic myelogenous leukemia treated with imatinib mesylate," International Journal of Hematology, vol. 81, no. 4, pp. 307-309, 2005.

[18] H. J. Kim, C. W. Jung, K. Kim et al., "Isolated blast crisis in CNS in a patient with chronic myelogenous leukemia maintaining major cytogenetic response after imatinib," Journal of Clinical Oncology, vol. 24, no. 24, pp. 4028-4029, 2006.

[19] A. Barlow, M. Robertson, A. Doig, W. Stewart, and M. W. Drummond, "Isolated central nervous system lymphoid blast crisis in chronic myeloid leukaemia in major molecular remission," British Journal of Haematology, vol. 142, no. 3, p. 327, 2008.

[20] K. W. Lee, M. K. Song, Y. M. Seol et al., "Isolated central nervous system blast crisis in chronic myeloid leukemia," The Korean Journal of Medicine, vol. 77, supplement 2, pp. S441-S444, 2009.

[21] A. Thomas, C. K. Stein, T. C. Gentile, and C. M. Shah, "Isolated CNS relapse of CML after bone marrow transplantation," Leukemia Research, vol. 34, no. 4, pp. el13-e114, 2010.

[22] M. Fuchs, M. Reinhöfer, A. Ragoschke-Schumm et al., "Isolated central nervous system relapse of chronic myeloid leukemia after allogeneic hematopoietic stem cell transplantation," $B M C$ Blood Disorders, vol. 12, article 9, 2012.

[23] S. G. O’Brien, F. Guilhot, R. A. Larson et al., "Imatinib compared with interferon and low-dose cytarabine for newly diagnosed chronic-phase chronic myeloid leukemia," The New England Journal of Medicine, vol. 348, no. 11, pp. 994-1004, 2003.

[24] B. J. Druker, F. Guilhot, S. G. O’Brien et al., "Five-year followup of patients receiving imatinib for chronic myeloid leukemia," The New England Journal of Medicine, vol. 355, no. 23, pp. 24082417, 2006.

[25] M. Deininger, S. G. O’Brien, F. Guilhot et al., "International randomized study of interferon vs. STI571 (IRIS) 8-year follow up: sustained survival and low risk for progression of events in patients with newly diagnosed chronic myeloid leukemia in chronic phase (CML-CP) treated with imatinib," Blood, vol. 114, no. 22, p. 462, 2009, Abstract 1126.
[26] J. F. Leis, D. E. Stepan, P. T. Curtin et al., "Central nervous system failure in patients with chronic myelogenous leukemia lymphoid blast crisis and Philadelphia chromosome positive acute lymphoblastic leukemia treated with imatinib (STI-571)," Leukemia \& Lymphoma, vol. 45, no. 4, pp. 695-698, 2004.

[27] H. Dai, P. Marbach, M. Lemaire, M. Hayes, and W. F. Elmquist, "Distribution of STI-571 to the brain is limited by P-glycoprotein-mediated efflux," Journal of Pharmacology and Experimental Therapeutics, vol. 304, no. 3, pp. 1085-1092, 2003.

[28] K. Porkka, P. Koskenvesa, T. Lundán et al., "Dasatinib crosses the blood-brain barrier and is an efficient therapy for central nervous system philadelphia chromosome positive leukemia," Blood, vol. 112, no. 4, pp. 1005-1012, 2008.

[29] T. O'Hare, D. K. Walters, E. P. Stoffregen et al., "In vitro activity of Bcr-Abl inhibitors AMN107 and BMS-354825 against clinically relevant imatinib-resistant Abl kinase domain mutants," Cancer Research, vol. 65, no. 11, pp. 4500-4505, 2005. 


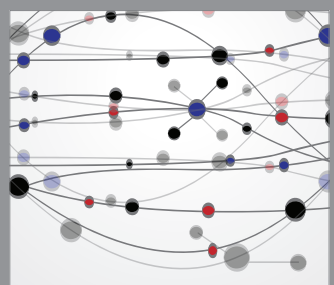

The Scientific World Journal
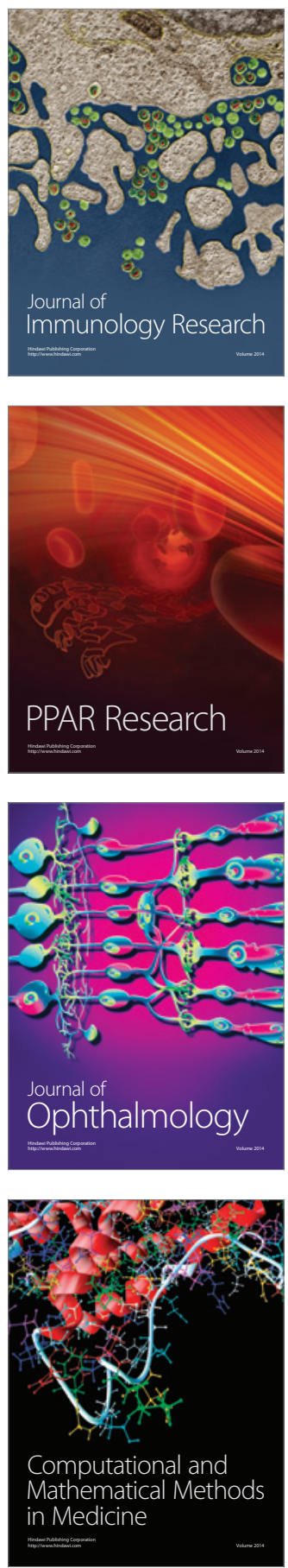

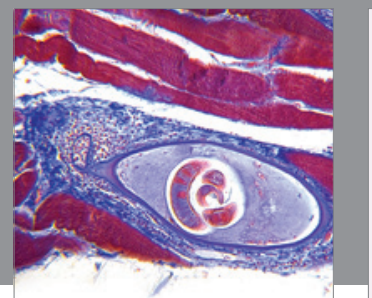

Gastroenterology

Research and Practice
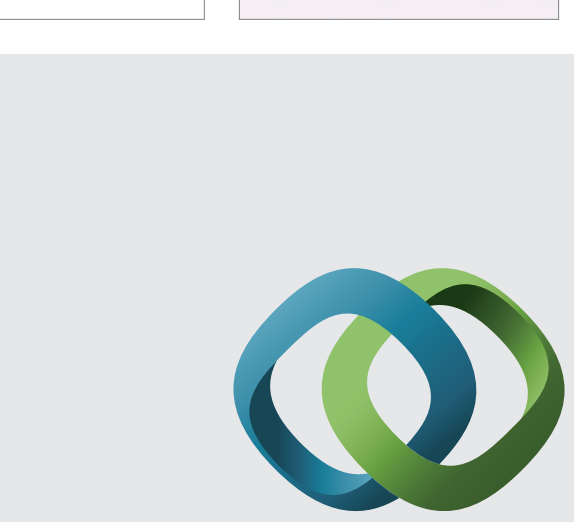

\section{Hindawi}

Submit your manuscripts at

http://www.hindawi.com
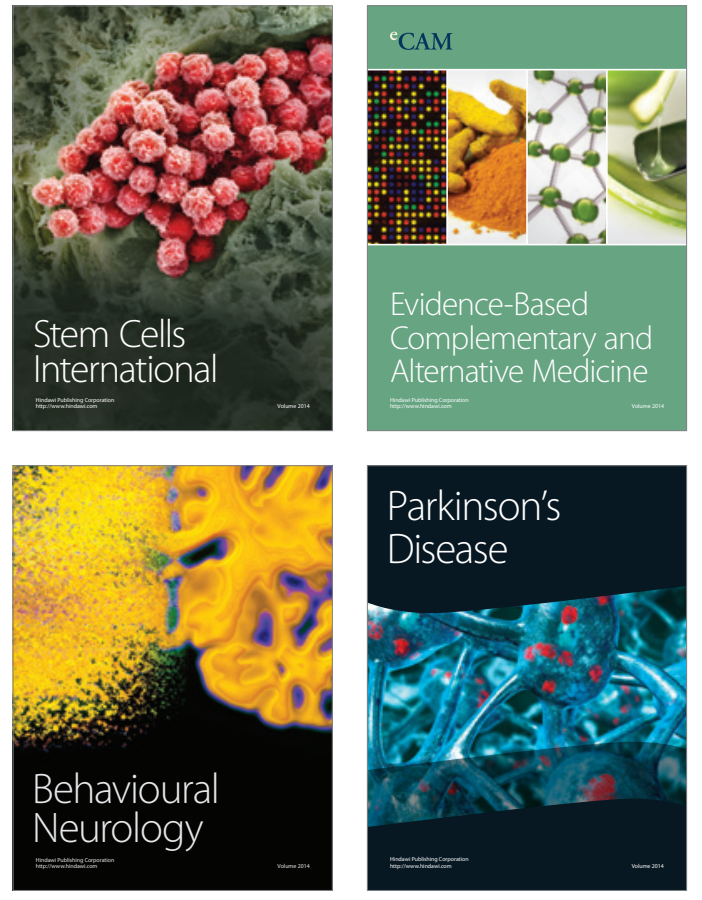
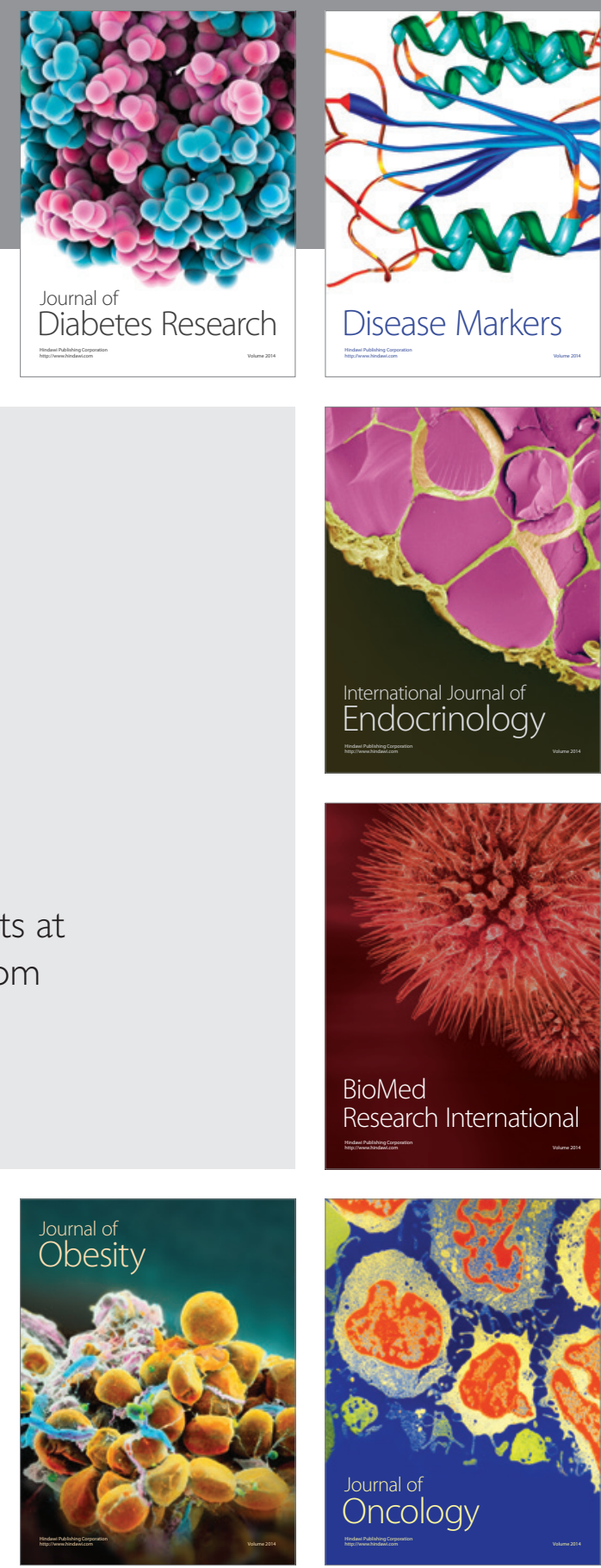

Disease Markers
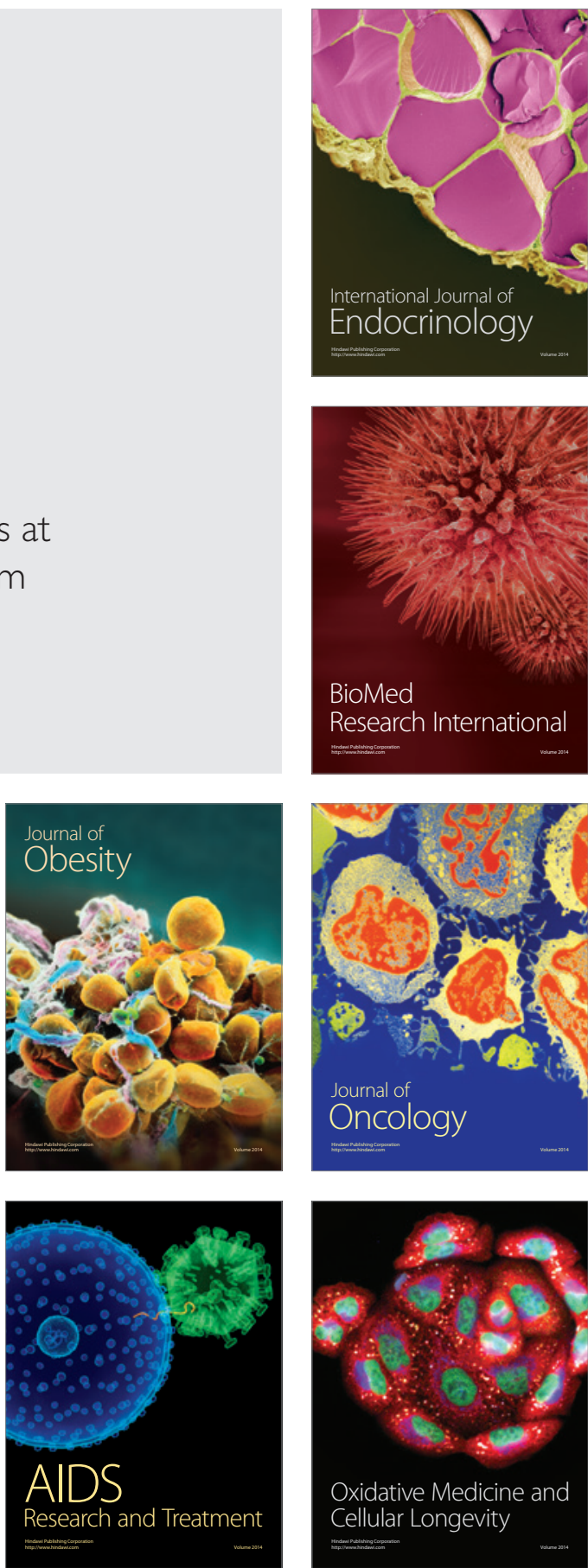